\title{
Poor shape perception is the reason reaches-to-grasp are visually guided online
}

\author{
Young-Lim LeE \\ Indiana University, Bloomington, Indiana \\ Charles E. Crabtree and J. Farley Norman \\ Western Kentucky University, Bowling Green, Kentucky \\ AND \\ Geoffrey P. Bingham \\ Indiana University, Bloomington, Indiana
}

\begin{abstract}
Both judgment studies and studies of feedforward reaching have shown that the visual perception of object distance, size, and shape are inaccurate. However, feedback has been shown to calibrate feedfoward reaches-tograsp to make them accurate with respect to object distance and size. We now investigate whether shape perception (in particular, the aspect ratio of object depth to width) can be calibrated in the context of reaches-to-grasp. We used cylindrical objects with elliptical cross-sections of varying eccentricity. Our participants reached to grasp the width or the depth of these objects with the index finger and thumb. The maximum grasp aperture and the terminal grasp aperture were used to evaluate perception. Both occur before the hand has contacted an object. In Experiments 1 and 2, we investigated whether perceived shape is recalibrated by distorted haptic feedback. Although somewhat equivocal, the results suggest that it is not. In Experiment 3, we tested the accuracy of feedforward grasping with respect to shape with haptic feedback to allow calibration. Grasping was inaccurate in ways comparable to findings in shape perception judgment studies. In Experiment 4, we hypothesized that online guidance is needed for accurate grasping. Participants reached to grasp either with or without vision of the hand. The result was that the former was accurate, whereas the latter was not. We conclude that shape perception is not calibrated by feedback from reaches-to-grasp and that online visual guidance is required for accurate grasping because shape perception is poor.
\end{abstract}

There is currently a puzzle in perception/action research on visually guided reaching and grasping. Many psychophysical studies have demonstrated that human observers cannot accurately perceive metric 3-D shape, but we ordinarily have no difficulty in reaching for and grasping solid objects. The puzzle is that accurate reaches-to-grasp would appear to require accurate shape perception, because grasping typically involves contact of the fingers with the back of an object. Grasps are known to be accurately sized relative to the size of an object as a hand approaches an object. When the grasp involves contact of thumb and fingers on the front and back of an object, respectively, then the specification of the relevant extent of the object (in depth) requires combined information about object size and shape. The shape can be characterized by the aspect ratio of object depth to width or by curvedness (Koenderink, 1990, pp. 319-324; Perotti, Todd, Lappin, \& Phillips, 1998). Specification of the metric object size in the frontoparallel plane together with the aspect ratio (or the curvedness) would determine the metric object depth. How can information about 3-D shape be determined ac- curately in order to permit accurate reaches-to-grasp? A solution is suggested by the collected results from studies on shape perception. One possible solution involves using feedback information from grasping to calibrate information about shape.

A large number of shape perception studies have investigated the relation between perceived shape and actual physical shape. Early studies began with single cues, such as binocular disparity or motion parallax. In monocular structure-from-motion studies (e.g., Norman \& Lappin, 1992; Norman \& Todd, 1993; Perotti et al., 1998; Tittle, Todd, Perotti, \& Norman, 1995; Todd \& Bressan, 1990; Todd \& Norman, 1991) and studies of stereopsis (e.g., Johnston, 1991; Tittle et al., 1995), the relative depth of objects has not been perceived accurately. Johnston examined perceived 3-D structure from binocular disparity and showed that shapes in depth tend to be systematically compressed at larger distances and systematically expanded at shorter distances. In other words, truly circular cylinders appeared flattened at a far distance and elongated at a near distance. Similar evidence for distortions in the percep-

G. P. Bingham, gbingham@indiana.edu 
tion of 3-D structure has been obtained in structure-frommotion studies. Tittle et al. (1995) asked observers to adjust the eccentricity of a motion-defined cylinder until its cross-section appeared circular. The observers consistently adjusted the cylinder so that its shape was compressed in depth. Because this compressed surface appeared cylindrical (i.e., it appeared to have a circular cross-section), this suggests that an expansion in depth occurs during perception. Unlike the earlier described stereoscopic distortion, the magnitude of the expansion was the same at different viewing distances. Todd and Bressan suggested that the relationship between physical and perceived space involved an affine distortion. They found that judgments of affine structure were accurate, whereas judgments of Euclidean (or metric) structure were not. These and other studies of perceived 3-D structure from binocular disparity and motion have shown that neither disparity alone nor motion alone is sufficient to perceive 3-D structure accurately and consistently over changes in viewing distance or orientation. When 3-D structure is presented in motion, the relation between physical and perceived structure appears to be affine distorted, but when 3-D structure is viewed stereoscopically, the affine properties appear to be systematically distorted as well.

Subsequent studies investigated the possibility that an accurate knowledge of 3-D structure might be derived from the combination of motion, binocular disparity, and other sources of optical information. However, Tittle et al. (1995) found inaccuracies in performance even when the 3-D objects were simultaneously defined by motion and binocular disparity. Adding yet further sources of information, such as lambertian shading, texture, and specular highlights also did not produce accurate perceptions of 3-D object shape (Norman \& Todd, 1996; Norman, Todd, $\&$ Phillips, 1995). Todd, Tittle, and Norman (1995) reviewed these results and concluded that "there is a nonEuclidean relationship between physical and perceived space, such that the true three-dimensional structures of objects appear systematically distorted" (p. 82).

Problems exist, however, with the idea that perception simply imposes a distortion, affine or otherwise, on perceived objects and space. For instance, if a circular cylinder in a structure-from-motion display were perceived to be stretched in depth (i.e., as a cylinder with an elongated elliptical cross-section), then, if this cylinder were rotated by $90^{\circ}$ around its symmetry axis, the rotation should be perceived as nonrigid because the cylinder would still be perceived to be elongated in depth after the rotation. However, such rotations are typically perceived as rigid despite the perceived constant expansion in depth and implied nonrigid shape change. Other results suggest that perceived depths might be merely ambiguous rather than systematically distorted. Todd and Norman (1991) found that observers could easily discriminate pairs of ellipsoids when they differed by a relative expansion along a frontoparallel axis, but could not discriminate pairs of ellipsoids when they were differentially stretched along the observers' line of sight. In this case, the objects were physically different in terms of 3-D shape but were perceived as the same. Todd and Norman (2003) asked ob- servers to adjust the depth of an object to match its width, the planes of dihedral angles to be orthogonal, and the shape of an object to match that of another presented at a different viewing distance. The results were variable and inconsistent with changes in viewing distance, orientation, or response task. In a similar vein, Lind, Bingham, and Forsell (2003) tested the perception of 3-D object shape while also varying viewing height and distance. The observers adjusted the shape of an elliptical outline on a computer screen to be the same as the perceived shape of elliptical cylinders (with a variety of depth-to-width ratios). The results differed across experiments and varied as a function of individual differences, differences in the range of shapes, and differences in the experimental tasks. The observers judged the object shapes correctly only when looking straight down on the tops of the objects (and could thus see the cross-sections or eccentricities of the cylinders). Lind et al. (2003) suggested that the shape of objects might be perceived incorrectly not because the objects' perceived shapes in depth are distorted, but because the perception of depth itself is ambiguous. Perotti et al. (1998) required observers to judge two different aspects of shape. One was the "shape index" (see Koenderink, 1990, pp. 319-324), which measures actual variations in surface shape (surface was cylindrical, ellipsoidal, shaped like a saddle, etc.). The other was "curvedness," which measures the amount or magnitude of surface curvature (Koenderink, 1990). Perotti et al. found that the shape index was judged accurately but that the judgments of curvedness were inaccurate and highly variable (see also Experiment 4 of Norman, Todd, Norman, Clayton, \& McBride, 2006).

On the one hand, the perception of 3-D shape has often been found to be systematically distorted. On the other hand, it seems that perceived shape is ambiguous rather than systematically distorted. If perceived shape is actually ambiguous, then the systematicity of the distortions found in many of the previously reviewed studies may have been produced by contextual factors. Lee, Lind, and Bingham (2007) investigated this hypothesis under full cue conditions, manipulating the relevant context by using two different ranges of object aspect ratios. Cylindrical objects were first presented so that the elliptical crosssections were frontoparallel (2-D task). These were judged accurately and provided a context for a second withinsubjects condition in which the objects were presented so that the elliptical cross-section occurred in depth (3-D task). The observers' task was to adjust the aspect ratio of a 2-D ellipse on a computer monitor to be the same as the cross-section of the target object. The 3-D judgments changed in predictable ways as a function of the context: A large range of 3-D aspect ratios following a small 2-D one yielded a decrease in the range of judged 3-D aspect ratios, whereas a small 3-D range following a large 2-D one yielded an increase in the range of judged 3-D aspect ratios. These results indicate that the perception of 3-D shape is ambiguous rather than systematically distorted and that the systematicity of perceptual distortions that is often obtained is produced by contextual variables specific to each individual study. 
This finding suggests a solution to the puzzle as to how accurate reaches-to-grasp might be performed despite inaccuracies in shape perception. Feedback information from grasping might be used to calibrate the perception of shape aspect ratios. That is, feedback information might play the role of a contextual variable both to resolve or constrain the ambiguity of perceived shape and to make it accurate enough for the perceptual control of reaches-tograsp. Previous studies have shown that accurate performance in visually guided reaching and grasping requires that participants use feedback to calibrate both reaches and grasps. Bingham and colleagues found that haptic feedback corrected the inaccuracy and instability of both reached distances and grasped object sizes during feedfoward reaching-to-grasp tasks (Bingham, 2005; Bingham, Coats, \& Mon-Williams, 2007; Bingham, Zaal, Robin, \& Shull, 2000; Coats, Bingham, \& Mon-Williams, in press; Mon-Williams \& Bingham, 2007). Also, Bingham et al. (2007) found calibration to be stable over a number of reaches-to-grasp. There was no difference in performance when feedback was presented during every trial versus only $50 \%$ of the trials. On the other hand, Bingham et al. (2000) found that the calibration does eventually drift. The perception of both distance (Mon-Williams \& Bingham, 2007) and size (Coats et al., in press) can be recalibrated by distorted haptic feedback from reaches-to-grasp. In those studies, the observers viewed target objects, without being able to see the hand with which they reached to grasp the target. However, during some trials, they actually grasped targets that either were at a different distance or possessed a different size from that of the target that they saw (although they were not aware of this). When the observers reached to grasp virtual target objects, they exhibited recalibration as a function of the distorted haptic feedback. The recalibration generalized over different distances and sizes of target objects.

The evidence shows that both object distance and object size can be calibrated to allow accurate perceptual guidance of reaches-to-grasp. However, this would not be sufficient for accurate grasping in most cases - namely, those in which the grasp involves an axis through an object that does not lie in a frontoparallel plane. In such cases, the accurate perception of object shape is also required. In fact, Bingham, Crowell, and Todd (2004) found that inaccuracies in distance perception and in shape perception are not produced by a single continuous transformation of perceived space. That is, they reflect different distortions or ambiguities. The implication is that they would require separate calibration if calibration is to enable accurate perception and performance. Bingham (2005) measured reaches to objects at different viewing distances to test whether distance, size, and shape perception are calibrated separately. Using a virtual environment system, reaches were measured in conditions with and without terminal feedback. In the condition without feedback, observers could not see their hands, although they could see the target object both before and during the reach. In the condition with feedback, observers could see a handheld stylus together with the target at the end of each reach. The results showed calibration of object distance and object (fronto- parallel) size with feedback, but there was no calibration of shape or object depth. Why was calibration of shape perception not elicited by feedback in this study, although calibration of distance and size perception was? There are at least three possibilities. The first is that the optics of the virtual environment may have been perturbing enough to destabilize shape perception and its calibration (although it did not interfere with distance or size perception and their calibration). This possibility should be retested in an environment with normal optics. Another reason might be that the action used in the study was reaching, not grasping. It may be that calibration is specific to the relevant action, which in this case would be grasping. The geometry and mechanics of the hand and fingers, in contrast to the arm, have been designed by evolution as a highly accurate positioning system. For instance, the motor mass (i.e., the muscle mass) is located off the hand on the arm to yield a high motor-mass-to-inertial-mass ratio. This is good for precision positioning. The calibration might be task specific, so we should test grasping itself. The third possibility is that perceived shape cannot be calibrated. In the present study, we investigated whether shape perception can be calibrated in an environment with normal optics by measuring grasp apertures during reaches-to-grasp, but before contact with an object. In Experiments 1 and 2 we tested the effect of distorted haptic feedback. In Experiment 3, we tested the effect of accurate haptic feedback. Finally, in Experiment 4, we investigated how effective grasping might occur if feedback failed to calibrate shape perception.

\section{EXPERIMENT 1}

In Experiment 1, we tested whether shape perception can be recalibrated via distorted haptic feedback by varying the target depth to manipulate the shape depth/width (D/W) aspect ratio. We designed two distorted feedback conditions. In the first condition, the aspect ratio was gradually decreased by decreasing object depth. In the second condition, the aspect ratio was gradually increased by increasing object depth. We measured the effect by using two different variables in three different types of trials. The two variables were the maximum grasp aperture (MGA), which occurs roughly halfway through a reach, and the terminal grasp aperture (TGA), which occurs at the end of a reach (reach velocity $\approx 0$ ) but before the fingers contact the target object (Bingham, Hughes, \& Mon-Williams, 2008; Coats et al., in press; Mon-Williams \& Bingham, 2005, 2008b; Mon-Williams, Coats, \& Bingham, 2004). The three trial types were feedback, probe, and test. In feedback trials, participants grasped an actual wooden target that was increasingly different in shape (e.g., elliptical) from the round cylindrical target that they saw. In probe trials, participants grasped a round cylindrical virtual target that they only saw. This was used to test the effect of the distorted calibration stimulus without the constraint of an actual object. This method has been used successfully in a number of studies (Bingham et al., 2007; Bingham \& Mon-Williams, 2004; Coats et al., in press; Coats, MonWilliams, \& Bingham, 2004; Mon-Williams et al., 2004; 
Mon-Williams \& Bingham, 2007, 2008a, 2008b; Sheehan, Bingham, \& Mon-Williams, 2008), in which it was also shown that awareness of distorted feedback did not prevent or interfere with recalibration. In test trials, participants grasped a virtual target that they only saw and that was different in shape from the round cylindrical target that was calibrated by haptic feedback and tested in probe trials. The goal of test trials was to test the generalization of recalibration to other shapes. If depth was gradually decreased (increased) in feedback trials, we used a test object with a large (small) depth to leave plenty of room for a recalibration effect. If calibrated with increased depth, then participants should reach to the small depth of the test object with an overly large grasp aperture. It was likely that participants would become aware that test trials involved only virtual objects because they involved the only nonround visible targets. However, such awareness (in addition to awareness of distorted feedback) had been shown in the previous studies not to affect performance.

\section{Method}

Participants. Twenty-four adults participated as observers. Twelve participated in one condition, and the remaining 12 participated in the other condition. All had normal or corrected-to-normal vision, and all were right-handed. All of the participants were naive as to the purpose of the study. The 14 participants at Indiana University were paid $\$ 7$ per hour and, the 10 participants at Western Kentucky University were given course credit. Half of the subjects at each university participated in each condition.

Apparatus. Participants sat near the corner of an L-shaped "mirror table" as shown in Figure 1. A semi-silvered mirror (which reflected $60 \%$ of the light and transmitted $40 \%$ ) was oriented $45^{\circ}$ to the line of sight extending across the corner of the L. The mirror was $33.7 \mathrm{~cm}$ wide $\times 24.3 \mathrm{~cm}$ high. A wooden surface $38 \mathrm{~cm}$ wide and $80 \mathrm{~cm}$ long was placed over each arm of the $\mathrm{L}$ at $20 \mathrm{~cm}$ below eye height. The surfaces were cut diagonally to fit the bottom edge of the mirror between the two surfaces. The L-shaped surface was painted matte black but was highly visible in normal room illumination. The participant's eye height was adjusted by changing seat height. The mirror was used to create the illusion that an object was behind the mirror, although it was actually located in front of the mirror. The rear surface of the mirror had a removable panel. When this was removed, the image of the object in front of the mirror could be perfectly aligned with a physically identical object behind the mirror. When the back panel of the mirror was replaced, the visible image of the object was the same as the physical object that could be grasped without vision of the hand. A black panel was placed at the front edge of the table in front of the participant. The upper edge of this panel dropped diagonally to the right so as to allow the bottom edge of the mirror to be visible while simultaneously occluding the participant's view of the surface and target in front of the mirror.

The targets were placed on the table surface to the left of the participant (i.e., in front of the mirror). The target objects were cylindrically shaped and painted matte black with green phosphorescent texture elements. All objects were $6.6 \mathrm{~cm}$ in width and $4.5 \mathrm{~cm}$ in height. Only the object depths were varied to manipulate the D/W aspect ratios. In feedback trials, participants saw a circular cylinder (diameter, $6.6 \mathrm{~cm}$ ) for which they received distorted haptic feedback. In probe trials, participants only saw (they could not touch) the round cylindrical virtual object. In test trials, participants only saw (they could not touch) an elliptical cylindrical virtual object. The shape of this test object was different in each of two conditions that also varied with respect to the distorted haptic feedback. In Condition 1 (increasing depth and aspect ratio), the feedback object depth was increased from $6.6 \mathrm{~cm}$ to $7.7 \mathrm{~cm}$, to $8.2 \mathrm{~cm}$, to $8.8 \mathrm{~cm}$ over four blocks of trials (one size per block), and the test object was $3.3 \mathrm{~cm}$ in

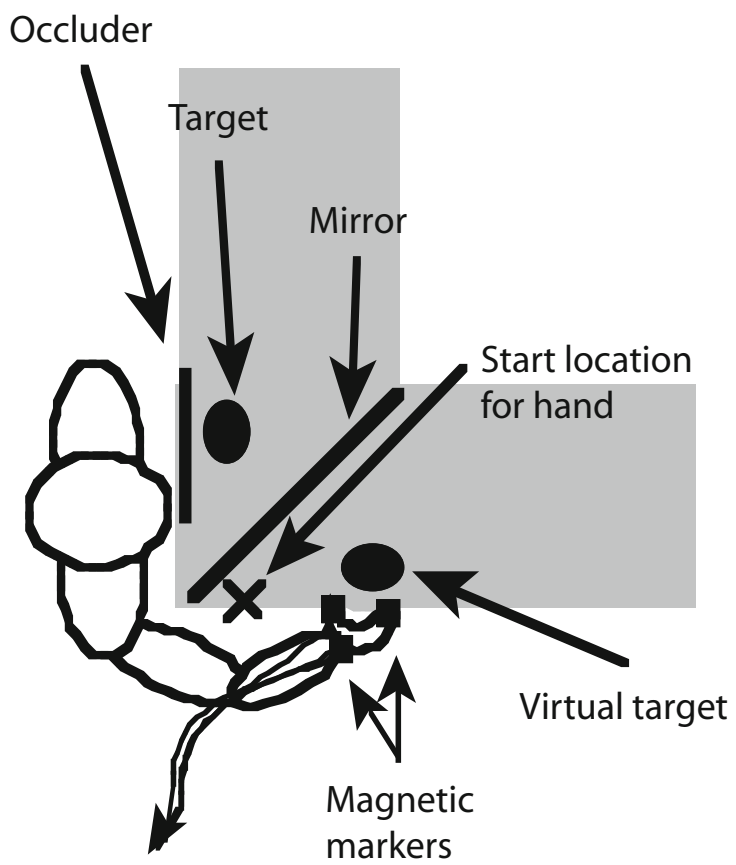

Figure 1. Arrangement of the experimental apparatus. See the text for explanation.

depth. In Condition 2 (decreasing depth and aspect ratio), the feedback object depth was decreased from $6.6 \mathrm{~cm}$ to $5.5 \mathrm{~cm}$, to $4.4 \mathrm{~cm}$, to $3.8 \mathrm{~cm}$ over four blocks of trials (one size per block), and the test object was $9.6 \mathrm{~cm}$ in depth. (See Figure 2.) To determine whether the recalibration generalized to other objects, we used a quite differently shaped object as a test object and one that left room for the proportional change in grasp aperture. An object that was small in depth was used when the depth of feedback objects was gradually increased, and an object that was large in depth was used when the depth of feedback objects was gradually decreased. If shape perception was recalibrated by the distorted haptic feedback, we expected that the aspect ratios derived from grasps of test objects should be altered in proportion to the distorted feedback. The targets were located near $(32 \mathrm{~cm})$ or far $(42 \mathrm{~cm})$ from the observer.

A three-marker Ascension miniBIRD magnetic measurement system was used to measure reach kinematics. This measurement system sampled movements at $103 \mathrm{~Hz}$. Markers $1.1 \times 0.8 \times 0.8 \mathrm{~cm}$ were placed on the nail of the index finger, the thumbnail, and the wrist of the right hand using double-sided tape. The wires were gathered around the forearm with tape. The emitter for the measurement system was located below the table and was centered in the reach space. Thick, black velveteen sound-attenuating drapes enclosed the experimental area around the L-shaped table and the participant. Black felt covered the table extending to the floor. The experimenter controlled the data collection using the computer keyboard, which was located beneath the surface. The data were stored in computer memory for subsequent analyses.

Procedure. The participants read and signed the consent forms, and then the markers were placed on the index finger nail, thumbnail, and wrist. The participant's eye height relative to the surface on which the target objects were placed was adjusted to $20 \mathrm{~cm}$ by changing the height of the chair. The task and procedure were explained. The participant sat at the table, pinched the index finger and thumb together, and then lightly rested them at the starting location (located at the right bottom corner of the mirror). The participant was asked to move from the start location to reach to grasp targets 


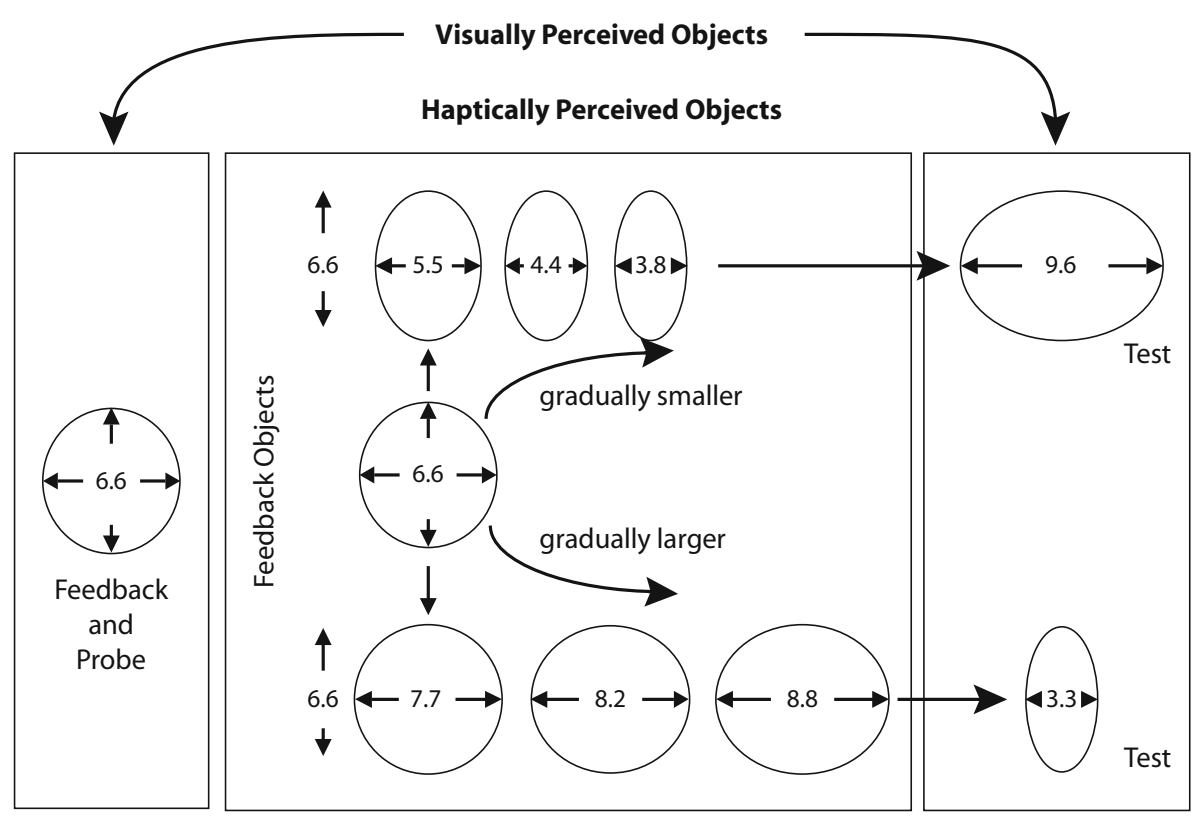

Figure 2. A schematic representation of the objects used in Experiments 1 and 2.

so as to span either the width or the depth with the index finger and thumb. The participant grasped object width and depth successively during a single target presentation. The participant reached to grasp the width, went back to the start position, and then reached to grasp the depth. The participant always grasped the target width first and then grasped the target depth. These successive grasps within a trial were used to compute an aspect ratio (depth to width). This was the measure of interest. The participants could see the targets in all trials, but they could not see their hands in any trial. They could touch targets in the feedback trials, but not in the probe or test trials.

There were two between-subjects conditions: Condition 1 involved decreasing object depth, and Condition 2 involved increasing object depth. Six probe trials occurred randomly among 6 feedback trials in each block of trials, with the constraint that the first trial in a block was always a feedback trial. The participants were not informed whether each trial would be feedback or probe (or test) during the experiment, although they were told that some trials would involve actual objects and others only virtual objects. Two test trials occurred at the end of a block of 12 feedback/probe trials. Four blocks of trials were tested. Each block was performed with a different increment of distorted haptic feedback, starting with the circular cylinder and proceeding through 3 increments. Two reaches (width then depth) were performed in 56 target presentations, for a total of 112 reaches. These methods, in which reaches to grasp physically present feedback objects were intermixed with reaches to grasp virtual objects (that were only visually present), were the same as those used in a number of previous studies. In particular, Bingham et al. (2007) showed that reaches to grasp virtual objects were normal and similar to reaches to grasp actual objects in all respects, as long as such reaches-to-grasp were calibrated by recent reaches to grasp actual objects.

The participants were asked to reach at a normal speed with the right hand and to grasp accurately. The participants' vision was occluded between trials while the target objects were placed in position. Once the target objects were placed, the participants' vision was restored. The experimenter then initiated data recording and verbally instructed the participants to reach to grasp the target object. Once the participants had reached and grasped the target, the experimenter stopped the data recording and told the participants to go back to the start position. The participants were informed that some target objects were virtual and that some were physically present. They were instructed that they should always reach to grasp what they saw. During feedback trials, if the participants failed to contact the feedback object, they were told to adjust their grasp in order to touch the object. The participants were not informed before the experiment (or during the experiment) that the feedback would be distorted. At the end of the experiment, the participants were debriefed and asked if they noticed anything odd and, if so, what they thought was happening and when it happened.

Dependent measures. To evaluate grasping with respect to the object shapes, the TGA and the MGA were used, as described earlier. Although the TGA occurs before contact of the hand with the target object, Mon-Williams and Bingham $(2005,2008 \mathrm{~b})$ found that it varies closely with object size. Typically, the grasp aperture at TGA is within $.5 \mathrm{~cm}$ of the object size. As described by Mon-Williams and Bingham (2005, 2008b), reaching-to-grasp is as much a collisionavoidance task as a target-acquisition task. The fingers are opened on approach to an object to avoid hitting the object as well as to prepare for the grasp. On approach, the variation in the orientation of the grasp aperture is as much a concern as is the variation in aperture size with respect to object size. As the orientation of the grasp aperture varies away from the horizontal (which does happen), the size of the grasp aperture has to be increased to avoid collision with the object. Mon-Williams and Bingham $(2005,2008 \mathrm{~b})$ found that the MGA is sized with respect to the maximum (diagonal) extent of the object or a diagonal formed by an approximately $45^{\circ}$ slice through the object, whichever is less. The MGA occurs at $50 \%-70 \%$ of the total duration and is identified simply as the maximum aperture size between the index finger and thumb. Thus, whereas the TGA varies with object size, Mon-Williams and Bingham (2005, 2008b) have shown that the MGA varies with the maximum object extent (MOE). MOE is computed as the Pythagorean of object width (or depth) and height - for instance, $\mathrm{MOE} \mathrm{W}=\left(\mathrm{W}^{2}+\mathrm{H}^{2}\right)^{5}$. Thus, the $\mathrm{D} / \mathrm{W}$ ratio computed using MOE was $\left(\mathrm{D}^{2}+\mathrm{H}^{2}\right)^{5} /\left(\mathrm{W}^{2}+\mathrm{H}^{2}\right)^{5}$. We used both the MGA and the TGA as dependent measures, because although the TGA is less variable than the MGA and varies closely with object size, the TGA could be constrained by the physical presence of the object. That is, the fingers might contact the objects at the TGA on 
some occasions. Nevertheless, we looked for redundant results using both measures; that is, they should replicate one another. If they did not, then we assumed that the physical presence of the object was interfering with the TGA. Since the markers were placed on the index finger nail and thumbnail, the thickness of the fingers was subtracted from the TGA and MGA. To measure the thickness of the fingers, the target object width or depth was subtracted from the final grasp aperture (FGA), which occurs when the fingers are finally in contact with the target object. Then, the thickness of the fingers was subtracted from the TGA and MGA to measure the actual distance between the two fingers.

\section{Results and Discussion}

In Experiment 1, we varied the target depth to investigate whether shape perception can be recalibrated by distorted haptic feedback. The results were somewhat equivocal, but two of the three measures showed that distorted haptic feedback did not recalibrate shape perception. There were no differences in the results for the targets at the two viewing distances (near and far), so we combined the data, ignoring this factor in other analyses. We computed the mean of all participants for each aspect ratio in each type of trial (feedback, probe, and test). In feedback trials, the aspect ratios of the TGA and MGA covaried with the aspect ratios of the feedback objects (see Figures 3 and 4). As the aspect ratio of the feedback object decreased (as the target depth decreased), the aspect ratios of the TGA and MGA also decreased. As the aspect ratio of the feedback object increased (as the target depth increased), the aspect ratios of the TGA and MGA also increased. However, the changes in the TGA aspect ratio and in the MGA aspect ratio were not significant for either the test or probe trials. (See Table 1.)

To evaluate the gain of the changes in aspect ratios, we computed the ratio between the rate of change (with respect to block number) of the aspect ratio of the feedback object and the rate of change of the TGA and MGA aspect ratios. The gains were computed as follows. Block number was regressed on TGA aspect ratios, MGA aspect ratios, or feedback object aspect ratios, respectively, to determine a slope in each case. Then, the TGA and MGA slopes were

Table 1

The Gains of the Terminal Grasp Aperture (TGA) Aspect Ratio and the Maximum Grasp Aperture (MGA) Aspect Ratio for Each Type of Trial (Feedback, Test, and Probe) in Experiment 1

\begin{tabular}{llrlrrr}
\hline & & \multicolumn{2}{c}{ TGA } & & \multicolumn{2}{c}{ MGA } \\
\cline { 3 - 4 } \cline { 6 - 7 } & & Gain & $r^{2}$ & & Gain & $r^{2}$ \\
\hline Condition 1 & Feedback & $80 \%$ & $.52^{* * *}$ & & $31 \%$ & $.04^{* * *}$ \\
& Test & $0 \%$ & .00 & & $0 \%$ & .00 \\
& Probe & $0 \%$ & .00 & & $0 \%$ & .02 \\
Condition 2 & Feedback & $99 \%$ & $.38^{* * *}$ & & $53 \%$ & $.06^{* * *}$ \\
& Test & $0 \%$ & .01 & & $0 \%$ & .01 \\
& Probe & $0 \%$ & .00 & & $0 \%$ & .03 \\
\hline
\end{tabular}

Note-In Condition 1 , the aspect ratio was gradually decreased by decreasing object depth. In Condition 2 , the aspect ratio was gradually increased by increasing object depth. Slopes were derived from regressions of block number on each measure, respectively (aspect ratios of TGA MGA, and actual feedback objects). To compute the gains, the slope of TGA and MGA aspect ratios were divided by the slope of the feedback object aspect ratio, which were -.14 and -.09 in Condition 1 , and .71 and .61 in Condition 2, respectively. The gains were set to $0 \%$ when the regressions failed to reach $p<.05 .{ }^{* * *} p<.001$. divided by feedback object slope to determine the relative or proportional response to the feedback changes. As is shown in Table 1, for the condition in which the aspect ratio was decreased by decreasing target depth, the gains of the TGA aspect ratio were $80 \%$ for the feedback trials, $0 \%$ for the test trials, and $0 \%$ for the probe trials. (When regressions were nonsignificant, slopes were set to 0 .) The gains of the MGA aspect ratio were $31 \%$ for the feedback trials, $0 \%$ for the test trials, and $0 \%$ for the probe trials. For the condition in which the aspect ratio was increased by increasing target depth, the gains of the TGA aspect ratio were $99 \%$ for the feedback trials, $0 \%$ for the test trials, and $0 \%$ for the probe trials. The gains of the MGA aspect ratio were $53 \%$ for the feedback trials, $0 \%$ for the test trials, and $0 \%$ for the probe trials. (See Table 1.)

The aspect ratios for probe and test trials did not change as a function of the distorted haptic feedback. We computed the mean values (computed over blocks) of these aspect ratios to compare them with those of the visible targets. As is shown in Figure 3, for the condition in which the aspect ratio was decreased by decreasing target depth, the overall mean TGA aspect ratio was .97 for probe trials and 1.23 for test trials as compared with the respective target aspect ratios of 1.00 and 1.45 . The overall mean MGA aspect ratio was .96 for probe trials and 1.15 for test trials as compared with 1.00 and 1.33 for targets (using ratios of MOEs). For the condition in which the aspect ratio was increased by increasing target depth, the overall mean TGA aspect ratio was .95 for probe trials and .56 for test trials as compared with 1.00 and .50 for targets. The overall mean MGA aspect ratio was .89 for probe trials and .64 for test trials as compared with 1.00 and .70 for targets (using ratios of MOEs). These aspect ratios were roughly in the ballpark of those for the objects viewed, but they were typically inaccurate (as will be shown subsequently in Experiment 3).

The results suggested, with some reservation, that perceived shape cannot be recalibrated by the haptic feedback experienced in reach-to-grasp tasks. Two out of three trial types failed to reflect recalibration-namely, the two virtual object trials, probe and test. These have reliably responded to calibration in previous studies of size and distance perception in reach-to-grasp tasks. Nevertheless, in the present study, the TGA and MGA did seem to reflect some recalibration in feedback trials. However, the physical presence of the objects may have constrained the grasping to yield this co-variation in the case of TGA. It was the MGA response in the feedback trials that suggested a possible weak calibration response. But without a result from the test trials, we had no real evidence for calibration; there was no evidence of generalization.

\section{EXPERIMENT 2}

In Experiment 1, we varied only the target depth. Previous studies have shown that the depth dimension is problematic for shape perception (Bingham, 2005; Johnston, 1991; Lind et al., 2003; Tittle et al., 1995; Todd \& Norman, 2003; Todd et al., 1995). In particular, Bingham found that 

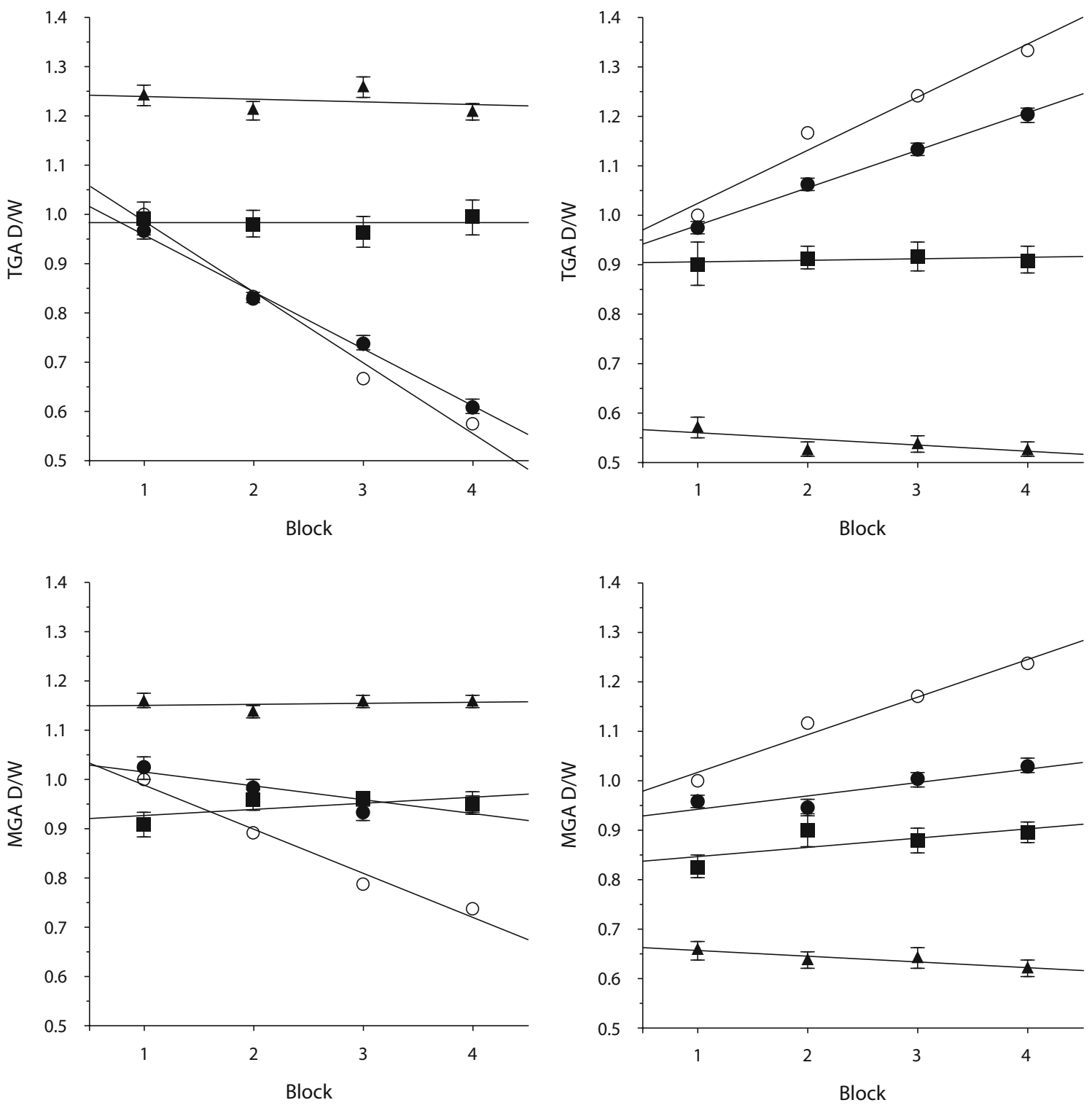

Figure 3. Mean TGA and MGA aspect ratios with standard error bars representing between-subjects variability in Experiment 1, Condition 1. In Condition 1, the target aspect ratio decreased by decreasing target depth. The upper panel shows the mean TGA aspect ratios, and the bottom panel shows the mean MGA aspect ratios, in both cases plotting the results of feedback, probe, and test trials across blocks. The visually specified aspect ratio for probe trials was 1 , and for the test trials it was 1.45 for TGA and 1.33 for MGA. Also shown are actual aspect ratios for feedback trials (open circles). The filled circles represent feedback trials, the filled squares represent probe trials, and the filled triangles represent test trials.

observers were able to calibrate object widths, but not object depths. We next tested whether shape perception can be recalibrated by distorted haptic feedback involving target width, instead of target depth. We tested two distorted

Figure 4. Mean TGA and MGA aspect ratios with standard error bars representing between-subjects variability in Experiment 1, Condition 2. In Condition 2, the target aspect ratio increased by increasing target depth. The upper panel shows the mean TGA aspect ratios, and the bottom panel shows the mean MGA aspect ratios, in both cases plotting the results of feedback, test, and probe trials across blocks. The visually specified aspect ratio for probe trials was 1 , and for test trials it was .5 for TGA and 7 for MGA.Also shown are actual aspect ratios for feedback trials (open circles). The filled circles represent feedback trials, the filled squares represent probe trials, and the filled triangles represent test trials.

feedback conditions. In the first condition, the aspect ratio was gradually decreased by increasing object width. In the second condition, the aspect ratio was gradually increased by decreasing object width. 


\section{Method}

Participants. Twenty-four adults participated as observers. Twelve participated in one condition, and the remaining 12 participated in the other. All had normal or corrected-to-normal vision, and all were right-handed. All of the participants were naive as to the purpose of the study. The 14 participants at Indiana University were paid \$7 per hour, and the 10 participants at Western Kentucky University were given course credit. Half of the subjects at each university participated in each condition.

Apparatus and Procedure. The apparatus and the procedure were the same as in Experiment 1, with one exception. The target shapes were varied by varying target width instead of depth. In Experiment 2, the target objects used in Experiment 1 were rotated by $90^{\circ}$ to present each major axis in a frontoparallel plane in order to vary target width. Each participant made 112 reaches in 56 target presentations.

\section{Results and Discussion}

Once again, the results were equivocal. The gains were computed as in Experiment 1. In Condition 1, the aspect ratio of the feedback object was gradually decreased. The aspect ratios of the TGA and of the MGA in feedback trials covaried with the aspect ratios of the feedback objects, as is shown in Figure 5. As the aspect ratio of the feedback object decreased (as target width increased), the aspect ratios of the TGA and of the MGA also decreased (with gains of $71 \%$ and $41 \%$, respectively). Changes in the TGA aspect ratio were significant for the test trials (gain $=78 \%$ ), but not for the probe trials (gain $=0 \%$ ). However, changes in the MGA aspect ratio were not significant for either the test or the probe trials (gain $=0 \%$ in both cases). (See Table 2.)

In Condition 2, the aspect ratio of the feedback object was gradually increased. In feedback trials, only the TGA aspect ratio, not the MGA aspect ratio, covaried with the aspect ratio of the feedback objects (gain $=64 \%$ ), as is shown in Figure 6. The changes in the TGA aspect ratio were also significant for probe trials (gain $=32 \%$ ) but not for test trials (gain $=0 \%$ ). No significant changes occurred in the MGA aspect ratios (gain $=0 \%$ in all cases). (See Table 2.)

As Figure 5 shows, for the condition in which the aspect ratio was decreased by increasing target width, the overall mean TGA aspect ratio was 96 for probe trials where there was no significant change as a function of feedback. This value compares well with 1.00 for the visual target object. The overall mean MGA aspect ratio was .92 for probe trials and 1.19 for test trials, neither of which changed with feedback. The latter does not compare well to the target value of 1.43. For the condition in which the aspect ratio was increased by decreasing target width, the overall mean TGA aspect ratio was .83 for test trials, which does not compare well to .69 for the visual target. The overall mean MGA aspect ratio was .95 for probe trials and .85 for test trials, which compares with 1.00 and .75 for the respective visual targets.

So, again the results were equivocal. Again, the TGA results in feedback trials could not be relied upon because of potential contamination from the actually present feedback object. This is why it was necessary to include reaches to grasp virtual objects. In Experiment 2, the distorted haptic feedback affected the MGA in only one of the two conditions. The probe trials reflected calibration
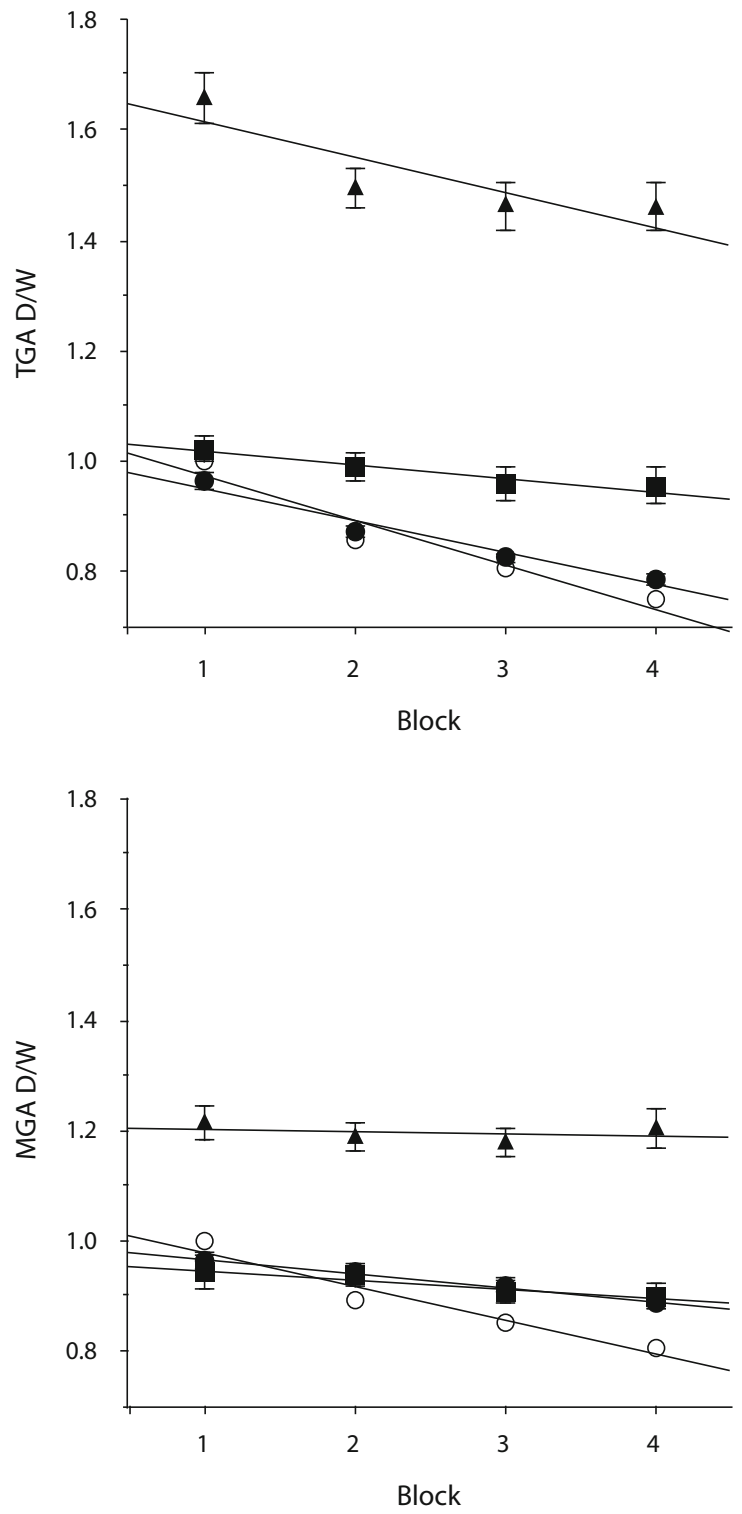

Figure 5. Mean TGA and MGA aspect ratios with standard error bars representing between-subjects variability in Experiment 2, Condition 1. In Condition 1, the target aspect ratio decreased by increasing target width. The upper panel shows the mean TGA aspect ratios, and the bottom panel shows the mean MGA aspect ratios, in both cases plotting the results of feedback, test, and probe trials across blocks. The visually specified aspect ratio for probe trials was 1 , and for test trials it was 2.0 for TGA and 1.43 for MGA. Also shown are actual aspect ratios for feedback trials (open circles). The filled circles represent feedback trials, the filled squares represent probe trials, and the filled triangles represent test trials.

in one condition and not the other, and, likewise, the test trials reflected calibration only in the opposite condition to that in which the probe trials did. This was the single instance out of a possible four in which we obtained any evidence for the generalization of calibration. These re- 
Table 2

The Gains of the Terminal Grasp Aperture (TGA) Aspect Ratio and the Maximum Grasp Aperture (MGA) Aspect Ratio for Each Type of Trial (Feedback, Test, and Probe) in Experiment 2

\begin{tabular}{llrllll}
\hline & & \multicolumn{2}{c}{ TGA } & & \multicolumn{2}{c}{ MGA } \\
\cline { 3 - 4 } \cline { 6 - 7 } & & Gain & $r^{2}$ & & Gain & $r^{2}$ \\
\hline Condition 1 & Feedback & $71 \%$ & $.32^{* * *}$ & & $41 \%$ & $.08^{* * *}$ \\
& Test & $78 \%$ & $.04^{* *}$ & & $0 \%$ & .00 \\
& Probe & $0 \%$ & .04 & & $0 \%$ & .02 \\
Condition 2 & Feedback & $64 \%$ & $.28^{* * *}$ & & $0 \%$ & .01 \\
& Test & $0 \%$ & .00 & & $0 \%$ & .00 \\
& Probe & $32 \%$ & $.15^{* * *}$ & & $0 \%$ & .01 \\
\hline
\end{tabular}

Note-In Condition 1, the aspect ratio was gradually decreased by increasing object width. In Condition 2 , the aspect ratio was gradually increased by decreasing object width. Slopes were derived from regressions of block number on each measure, respectively (aspect ratios of TGA, MGA, and actual feedback objects). To compute the gains, the slope of TGA and MGA aspect ratios were divided by the slope of the feedback object aspect ratio, which were -.08 and -.06 in Condition 1 , and .25 and .12 in Condition 2, respectively. The gains were set to $0 \%$ when the regressions failed to reach $p<.05 .{ }^{* *} p<.01 .{ }^{* * * *} p<.001$.

sults contrast strongly with those from previous studies of calibration (of size and distance) where strong and consistent results were obtained.

\section{EXPERIMENT 3}

In Experiments 1 and 2, the results were equivocal. Some measures seemed to show calibration, but others did not. Test trials designed to test the generalization of calibration exhibited evidence of such generalization in only one out of four cases. What should one conclude from this? The point of calibration is to allow accurate reaching-to-grasp despite instabilities and inaccuracy in space perception - in this case, the perception of metric shape. A common saying is that "the proof of the pudding is in the eating." So, the ultimate test should consist of reaching-to-grasp with accurate haptic feedback. If this feedback would in fact calibrate otherwise inaccurate shape perception, then feedforward reaches-to-grasp with intermittent (correct) feedback should yield accurate shape, reflected in the grasps. If not, then the results should replicate those from comparable judgment studies of shape perception. In fact, such judgment studies have been performed with the same objects that were used in the present experiments (Lee, Lind, \& Bingham, 2007, 2008; Lind et al., 2003). We will compare the present results with the previous results from judgment studies.

\section{Method}

Participants. Twenty adults participated as observers. All had normal or corrected-to-normal vision and were right-handed. All of the participants were naive as to the purpose of the study. The 10 participants at Indiana University were paid $\$ 7$ per hour, and the 10 participants at Western Kentucky University were given course credit.

Apparatus and Procedure. The apparatus was the same as in the previous experiments. Three different object shapes were used for this experiment. The medium object had a circular shape $6.6 \mathrm{~cm}$ in diameter. The small object had an elliptical shape $3.3 \times 6.6 \mathrm{~cm}$, and the large object had an elliptical shape $6.8 \times 9.6 \mathrm{~cm}$. All objects were $4.5 \mathrm{~cm}$ in height. The objects were rotated by $90^{\circ}$ to present each major axis in a frontoparallel plane, so that the three different object shapes yielded five different object aspect ratios (Figure 7). The targets were located near to $(32 \mathrm{~cm})$ or far from $(42 \mathrm{~cm})$ the observer.
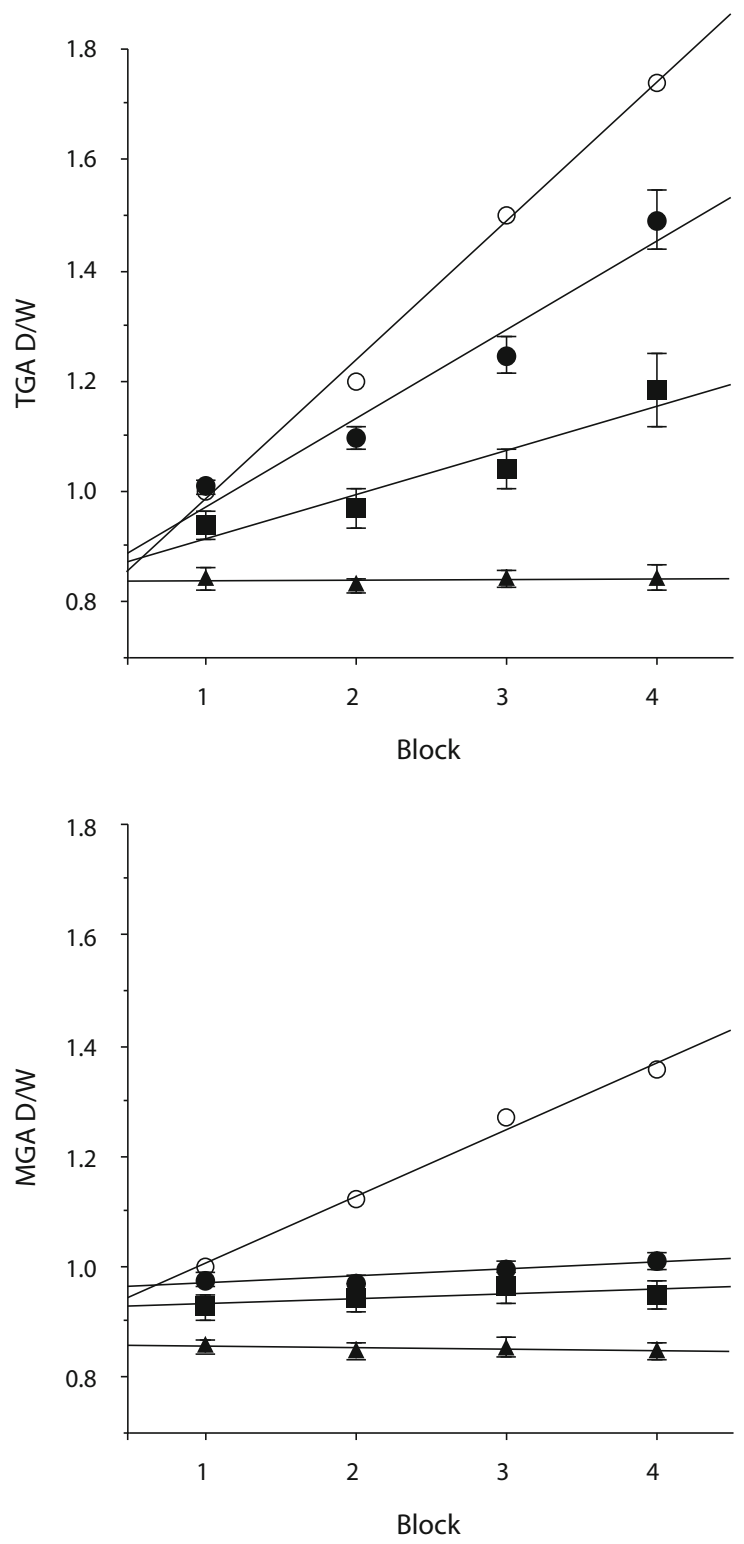

Figure 6. Mean TGA and MGA aspect ratios with standard error bars representing between-subjects variability in Experiment 2, Condition 2. In Condition 2, the target aspect ratio increased by decreasing target width. The upper panel shows the mean TGA aspect ratios, and the bottom panel shows the mean MGA aspect ratios, in both cases plotting the results of feedback, test, and probe trials across blocks. The visually specified aspect ratio for probe trials was 1 , and for test trials it was .69 for TGA and $\mathbf{7 5}$ for MGA. Also shown are actual aspect ratios for feedback trials (open circles). The filled circles represent feedback trials, the filled squares represent probe trials, and the filled triangles represent test trials. 


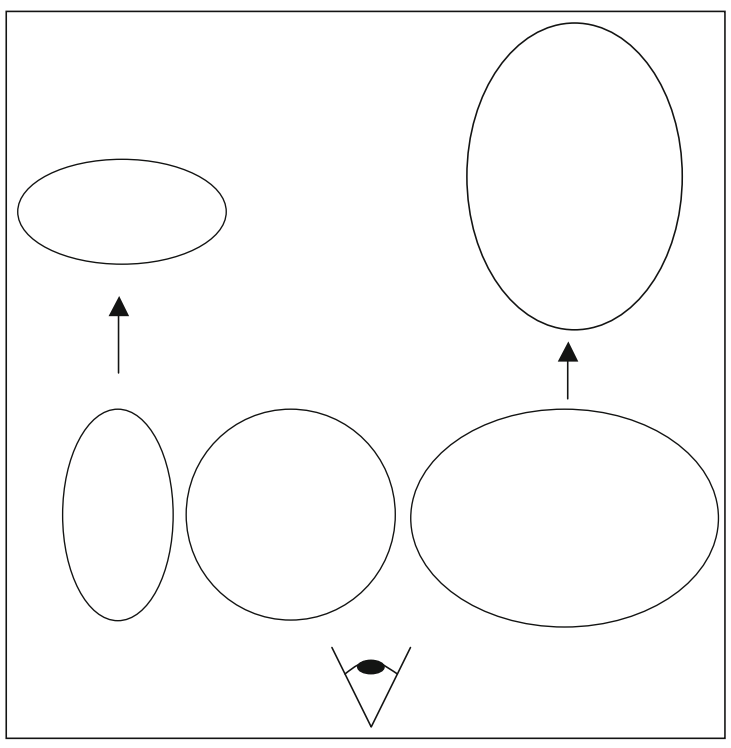

Figure 7. A schematic representation of the objects used in Experiments 3 and 4 .

During feedback trials, the participants could both see and touch the object, but could not see their hand. The participants received correct haptic feedback. They touched an object that was identical to the one that they saw. During probe trials, there were no feedback objects (i.e., strictly virtual targets). This was demonstrated during instruction before the start of the experiment, but the participants were not informed whether each trial would be probe or feedback during the experiment. The participants were allowed to practice reaching to grasp a circular object width at a near distance before the start of the experiment. After a few practice trials, the participants reached to grasp width, went back to the start position, and then reached to grasp depth. All aspect ratio-distance combinations $(5 \times 2)$ were presented in the first 10 trials, which were feedback trials. During the remaining 40 trials, probe and feedback trials were randomly intermixed, with 20 of each, the 20 consisting of two repetitions of each aspect ratio-distance combination. The participants performed 100 reaches (reaches to grasp object width, then depth) in 50 trials.

\section{Results and Discussion}

In Experiment 3, we investigated whether TGA and MGA aspect ratios covaried with the target aspect ratio in feedback and probe trials. Results showed that the TGA aspect ratio varied reliably and accurately with the target aspect ratio in feedback trials, but both over- and underestimated aspect ratios in probe trials: In a regression of target aspect ratios on TGA aspect ratios, the slope was near 1 for feedback trials, but significantly less than 1 for probe trials. For the MGA, the slope was significantly less than 1 for both feedback and probe trials. The TGA aspect ratios for feedback trials were likely contaminated by the presence of the actual objects. Thus, we must focus on the TGA results for probe trials and the MGA results. These results compare well with those obtained in judgment studies, meaning that perceived metric shape is not reliably accurate. Feedforward reaching does not reflect the accurate calibration of metric shape.
We computed both the slope of the relation between target aspect ratios and TGA aspect ratios and the slope of the relation between target $\mathrm{MOE}$ aspect ratios and MGA aspect ratios. A slope was computed for the means of each participant, combining the data at the two target distances. In the feedback trials, the slope for the TGA aspect ratio was .93 and the $r^{2}$ was .96 . In the probe trials, the slope for the TGA aspect ratio was .72 and the $r^{2}$ was .84. (See Figure 8.) In the feedback trials, the slope for the MGA aspect ratio was .76 and the $r^{2}$ was .76 . In the probe trials, the slope for the MGA aspect ratio was .6 and the $r^{2}$ was .63. (See Figure 9.)
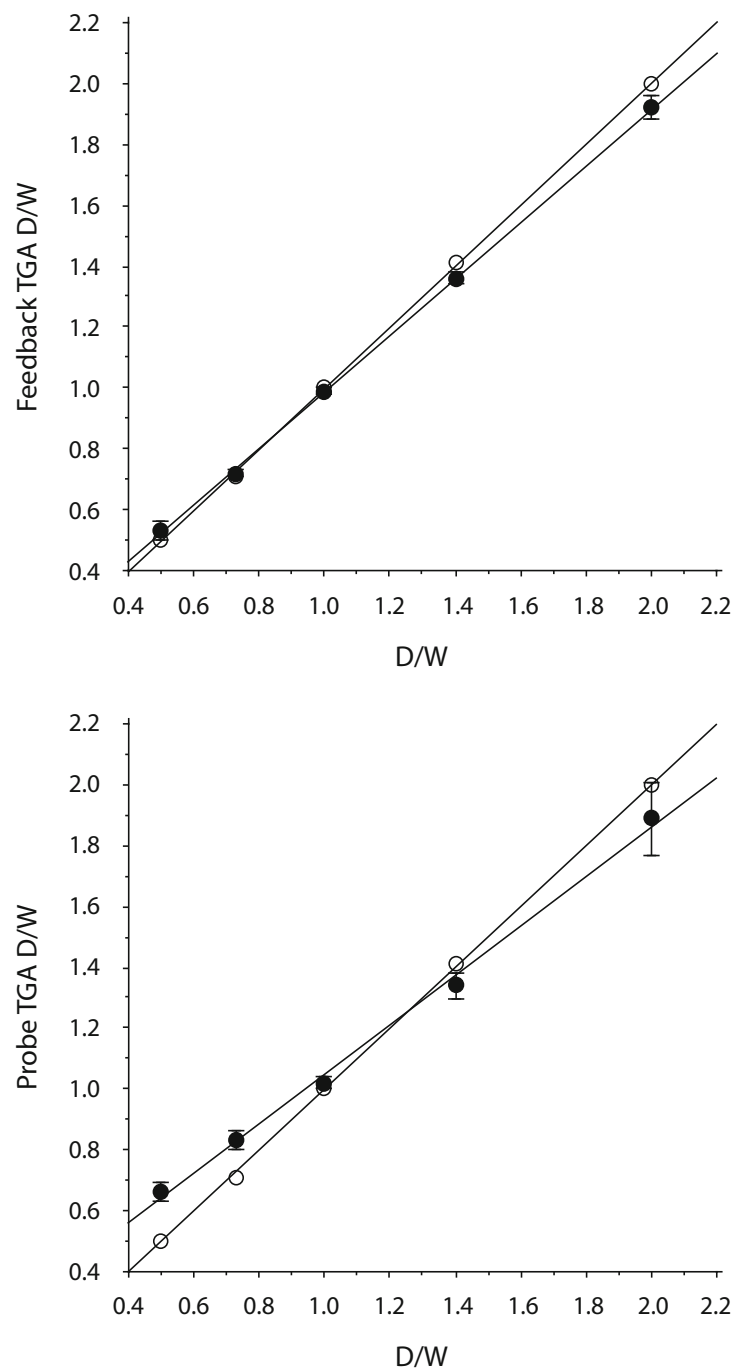

Figure 8. Mean TGA aspect ratios with standard error bars representing between-subjects variability in Experiment 3. The upper panel shows the mean TGA aspect ratios in feedback trials, and the bottom panel shows the mean TGA aspect ratios in probe trials as a function of actual target aspect ratio. The filled circles represent the judged aspect ratios, and the open circles represent the correct target aspect ratio. 

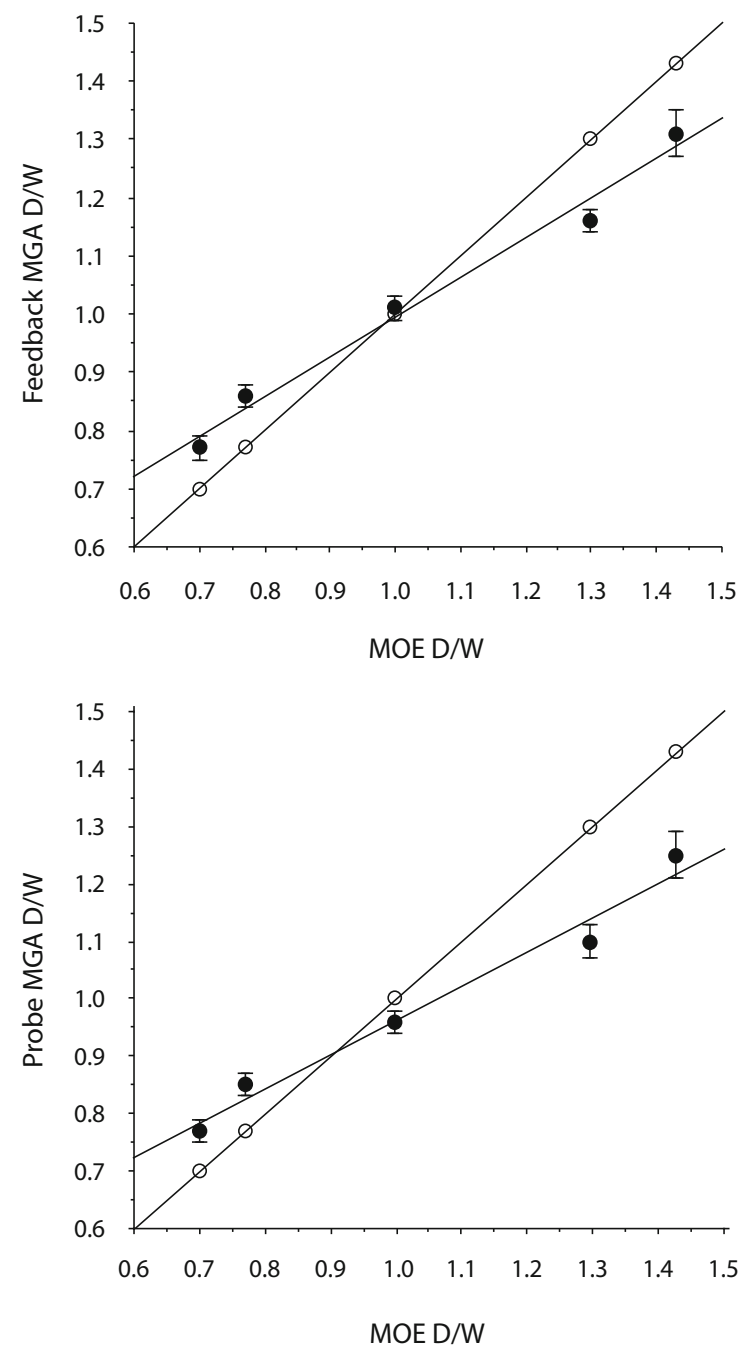

Figure 9. Mean MGA aspect ratios with standard error bars representing between-subjects variability in Experiment 3. The upper panel shows the mean MGA aspect ratios in feedback trials, and the bottom panel shows the mean MGA aspect ratios in probe trials as a function of actual target MOE aspect ratio. The filled circles represent the judged aspect ratios, and the open circles represent the correct target aspect ratio.

As can be seen in Figure 9, there was no difference between feedback and probe trials for the MGA aspect ratios. The small target aspect ratios were overestimated and the large target aspect ratios were underestimated in both the feedback and probe trials. However, the pattern of the TGA aspect ratio in feedback trials was different from the pattern obtained in probe trials shown in Figure 8. The TGA aspect ratios in probe trials showed the same pattern as did the MGA aspect ratios in probe and feedback trials. Clearly, the TGA must have been affected by the actual object presence in the feedback trials. The pattern of the MGA in both the probe and feedback trials was replicated by the TGA in probe trials.
To confirm these results, we performed multiple regressions to test differences in slopes between conditions separately for the TGA and MGA data. There were three independent variables: target aspect ratio (computed with MOEs for MGA analysis), feedback versus probe trials (coded as \pm 1 ), and an interaction vector (computed as the product of the first two vectors) (Pedhazur, 1982). The dependent measures were TGA or MGA aspect ratios. There was a main effect of trial type in the analysis on TGA aspect ratios [partial $F(3,996)=4.12, p<.001]$, as well as a significant interaction [partial $F(3,996)=3.55$, $p<.001$ ] showing that there was a significant difference in slope between the feedback and probe trials. However, there was neither a significant main effect nor a significant interaction in the analysis on the MGA aspect ratios.

We compared these results with those obtained in previous judgment studies of shape perception that were performed with the same objects as those used in the present experiments. Lind et al. (2003) performed three experiments in which they tested different ranges of aspect ratios. The participants viewed the objects in conditions comparable to those of the present experiments, but without the mirror apparatus. The judgments of metric shape were performed either by adjusting an elliptical outline on a computer monitor to be the same as the perceived shape of the top of the cylindrical objects or by picking objects from a large set of comparison objects (viewed by looking straight down at the top of the objects that were, therefore, viewed in a frontoparallel plane) to match target objects viewed in 3-D perspective. (We have performed many such studies, all with comparable results, only some of which were published in Lind et al. [2003].) The slope of the relation between actual and judged aspect ratios varied, depending on the range of aspect ratios tested. The slope for the experiment with a range comparable to that for the present experiments was .72. This is the same as that found in the present results. Other judgment studies testing different ranges yielded slopes that were comparably greater than 1 -for example, slopes of 1.37 or 1.23 . As has been shown by Lee et al. $(2007,2008)$, these values can vary as a function of contextual variables in judgment studies. Nevertheless, we can conclude in the present case that these reach-to-grasp results are comparable to those of previous judgment studies that demonstrated an inaccurate perception of metric shape. Thus, our results show that feedforward grasping is poorly guided with respect to object shape. Although size and distance perception can be calibrated by haptic feedback (Bingham, 2005; Bingham et al., 2000; Coats et al., in press; Mon-Williams \& Bingham, 2007), shape perception is not well calibrated by haptic feedback without vision of the hand. This leaves the obvious question: How is effective (i.e., accurate) grasping achieved? Also, is it possible to find MGA and TGA results that reflect accurate performance with regard to metric shape?

\section{EXPERIMENT 4}

In Experiment 3, we concluded that haptic feedback without vision of the hand cannot correct the inaccuracy 
of perceived shape as reflected in feedforward grasping behavior. However, we normally reach to grasp very well. How do we normally perform effective grasping? We hypothesized that we normally perform effective grasps by using online guidance (closed-loop control) to gear hand preshape with respect to object shape, and that poor shape perception is essentially the reason why such online guidance is needed. In Experiment 4, we compared grasping performance with and without online guidance.

\section{Method}

Participants. Forty adults participated in each of two experimental conditions (20 participated in one condition, and the remaining 20 participated in the other condition). All had normal or correctedto-normal vision, and all were right-handed. All of the participants were naive as to the purpose of the study. The 20 participants at Indiana University were paid $\$ 7$ per hour, and the 20 participants at Western Kentucky University were given course credit. Half of the subjects at each university participated in each condition. However, we did not use the data of 10 Indiana participants who performed in Condition 2, because the three-marker Ascension miniBIRD magnetic measurement system did not function well when we collected data in Condition 2.

Apparatus and Procedure. The apparatus was the same as in the previous experiments. The procedure was the same as in Experiment 3, with two exceptions. First, there were no probe trials in Experiment 4 . Second, width and depth were grasped separately in the blocked presentations, instead of successive grasping of width and then depth in a single target presentation. There were two betweensubjects conditions. In Condition 1 (closed-loop control), the back panel of the mirror was removed, so that the participants could see their hand. In Condition 2 (open-loop control), the back panel of the mirror was replaced, so that the participants' view of their hand was occluded. In both conditions, participants could both touch and see targets. In each condition, the widths were tested first in five blocks of repeated trials, followed by five blocks in which depths were grasped. In each block, all five aspect ratios were presented at each of the two distances in a random order. Each participant was tested on a total of 100 trials ( 2 directions $\times 5$ aspect ratios $\times 5$ repetitions $\times 2$ distances)

\section{Results and Discussion}

In Experiment 4, we investigated whether there is a difference between open-loop control and closed-loop control during reaches-to-grasp. The results supported our hypothesis that closed-loop control is needed for effective grasping. In Condition 1 (closed-loop control), the TGA and MGA aspect ratios were generally accurate, but in Condition 2 (open-loop control), the TGA and MGA aspect ratios were too large for small target aspect ratios and too small for large target aspect ratios: that is, the slope of the relation between actual aspect ratios and those derived from grasps was significantly less than 1 .

We computed both the slope of the relation between the TGA aspect ratio and the target aspect ratio and the slope of the relation between the MGA aspect ratio and the MOE aspect ratio in each condition. A slope was computed using the means of each participant, combining the data at the two target distances. In Condition 1 (closedloop control), the slope for the TGA aspect ratio was .97 and the $r^{2}$ was .97. In Condition 2 (open-loop control), the slope for the TGA aspect ratio was .83 and the $r^{2}$ was .96 . (See Figure 10.) In Condition 1 (closed-loop control), the
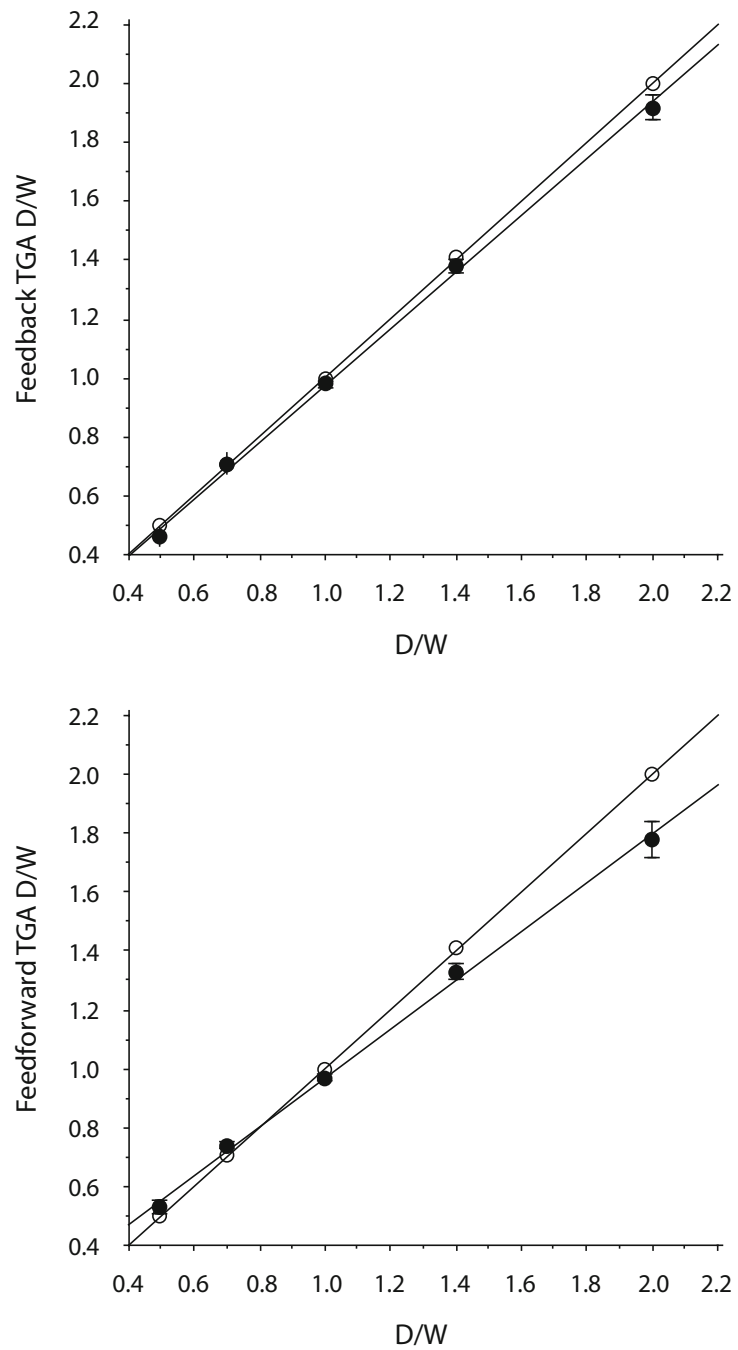

Figure 10. Mean TGA aspect ratios with standard error bars representing between-subjects variability in Experiment 4 . The upper panel shows the mean TGA aspect ratios in Condition 1 (closed-loop control), and the bottom panel shows the mean TGA aspect ratios in Condition 2 (open-loop control) as a function of actual target aspect ratio. The filled circles represent the judged aspect ratios, and the open circles represent the correct target aspect ratio.

slope for the MGA aspect ratio was 1.15 and the $r^{2}$ was .87. In Condition 2 (open-loop control), the slope for the MGA aspect ratio was .61 and the $r^{2}$ was .86. (See Figure 11.) The closed-loop results were different from the results in Experiment 3, but the open-loop results were comparable. Perceived metric shape, as reflected in MGA behavior, was accurate only in the case of closed-loop reaching-to-grasp. This is important. It shows that MGA can vary accurately with object shape and that, therefore, when it does not (viz., under conditions of feedforward control), we can infer that this is because object shape is not perceived accurately. 

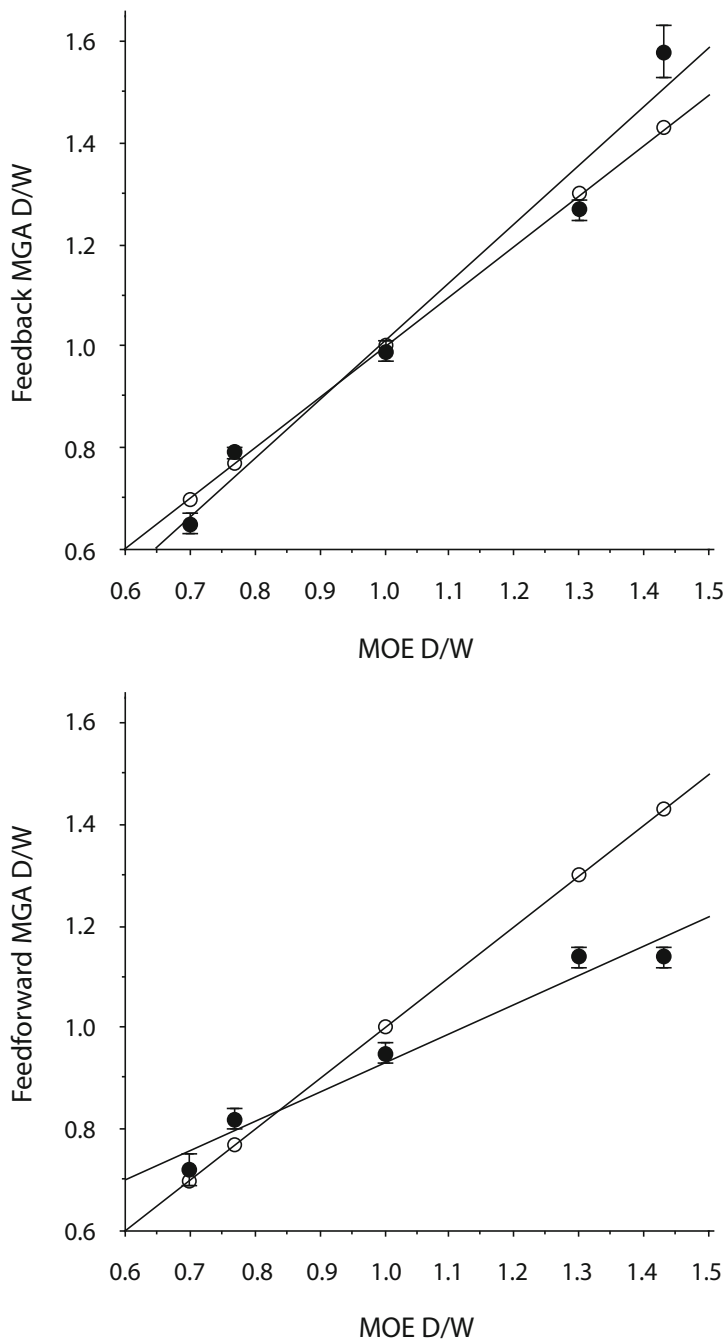

Figure 11. Mean MGA aspect ratios with standard error bars representing between-subjects variability in Experiment 4 . The upper panel shows the mean MGA aspect ratios in Condition 1 (closed-loop control), and the bottom panel shows the mean MGA aspect ratios in Condition 2 (open-loop control) as a function of actual target aspect ratio. The filled circles represent the judged aspect ratios, and the open circles represent the correct target aspect ratio.

We performed multiple regressions to test differences in slopes between conditions separately for the TGA and MGA data. There were three independent variables: target aspect ratio (computed with MOEs for MGA analysis), closed-loop versus open-loop trials (coded as \pm 1 ), and an interaction vector (computed as the product of the first two vectors) (Pedhazur, 1982). The dependent measures were TGA or MGA aspect ratios. There was a main effect of trial type in the analysis on TGA aspect ratios [partial $F(3,146)=3.82, p<.001]$, as well as a significant interaction [partial $F(3,146)=4.85, p<.001]$ showing that there was a difference in TGA slope between closed-loop and open-loop conditions. Furthermore, there was a main effect of trial type in the analysis on MGA aspect ratios [partial $F(3,146)=6.29, p<.001$ ], as well as a significant interaction [partial $F(3,146)=7.87, p<.001$ ] showing that there was a difference in MGA slope between the closed-loop and open-loop conditions. These results show that accurate and effective grasping requires online guidance (closed-loop control).

In summary, the TGA and the MGA aspect ratios varied reliably and accurately with the target aspect ratios in Condition 1 (closed-loop control). However, the small target aspect ratios were overestimated while the large target aspect ratios were underestimated in Condition 2 (open-loop control). We conclude that online guidance (closed-loop control) is needed for effective and accurate grasping. In fact, poor shape perception appears to be the reason why reaches-to-grasp need to be continuously guided.

\section{GENERAL DISCUSSION}

A large number of shape perception studies have shown that metric 3-D shape cannot be perceived accurately and that the perception of object shape is frequently distorted (see Todd et al.'s 1995 review). The recovery of 3-D structure is inaccurate in the perception of structure from motion (Norman \& Lappin, 1992; Norman \& Todd, 1993; Perotti et al., 1998; Tittle et al., 1995; Todd \& Bressan, 1990; Todd \& Norman, 1991), the perception of structure from binocular disparity (Johnston, 1991; Tittle et al., 1995), the perception of structure from the combination of binocular disparity and motion (Tittle \& Braunstein, 1993; Tittle et al., 1995), and even the perception of structure under relatively full cue conditions (Norman \& Todd, 1996; Norman et al., 1995). It has been suggested, however, that the inaccurate perception of 3-D object shape might occur not because of systematic distortion, but because of ambiguity (Lind et al., 2003; Todd \& Norman, 2003), and that the systematicity of perceptual distortions is produced by contextual variables (Lee et al., 2008).

The poor shape perception previously found in shape perception studies is perplexing in the context of visually guided reaching and grasping because we normally perform reaches-to-grasp well and one would think that accurate shape perception is required for accurate reachesto-grasp. Object size and shape information should be combined for accurate grasping when the grasp involves contact of thumb and fingers on the front and back of an object, as it so often does. Thus, the question is, How can we perform reaches-to-grasp well, despite poor shape perception? One possible solution is that feedback information might constrain the ambiguity of perceived shape and calibrate information about shape so as to allow accurate reaches-to-grasp.

In Experiment 1, we tested whether shape perception is recalibrated by distorted haptic feedback. Mon-Williams and Bingham (2007) and Coats et al. (in press) found that observers recalibrated distance and size as a function of distorted haptic feedback. In Experiment 1, we varied the target depth to manipulate the aspect ratio of target objects. We gradually increased the aspect ratio by increasing the depth in one condition and decreased the aspect ratio by 
decreasing the depth in another condition. The observers viewed the target objects, but were not able to see their hand during reaching to grasp the targets. During some trials, they actually grasped targets with a different aspect ratio than what they saw, and during other trials, they grasped virtual targets without touching them. We found that recalibration did not occur when observers reached to grasp virtual objects. Similarly, in Experiment 2, we varied target width instead of target depth, and the results were equivocal when observers reached to grasp virtual objects. It is likely that no effects on reaches to grasp virtual targets were found in Experiment 1 because the depth varied in feedback and the perception of object depth is so problematic. In Experiment 2, we varied shape aspect ratios by varying only the object widths, and these can be calibrated if only the perception of object size is at issue. We may have obtained some (inconsistent) effects in reaches to grasp virtual objects for this reason.

The first two experiments suggested that perceived shape is not reliably recalibrated by distorted haptic feedback, although previous studies have shown that perceived distance and size can be recalibrated by such feedback. This result is consistent with the results of Bingham (2005) in which shape perception was not calibrated by haptic feedback but distance and size perception were. These results indicated that effective feedforward grasping is not based on the calibration of shape perception, although the results were somewhat equivocal. So, we next investigated the accuracy of feedforward grasping with respect to object shape in the context of accurate feedback that would allow reaches-to-grasp to be calibrated and accurate. In Experiment 3, observers were able to see a target object and touch an object that matched what they saw, but they were not able to see their hand. In addition, we tested the possibility that the physical presence of the object might constrain the TGA by adding random probe trials. During the probe trials, the observers did not touch the object, although they were able to see the object (i.e., virtual targets). The results for the feedback trials showed that the patterns of the TGA and the MGA aspect ratios were different. The TGA aspect ratio was close to the actual target aspect ratio, but the MGA aspect ratio was not. On the other hand, the results for the probe trials showed that the patterns of the TGA and the MGA aspect ratios were the same and were the same as the MGA aspect ratios obtained for feedback trials. In the latter three cases, the small target aspect ratios were overestimated and the large target aspect ratios were underestimated. Thus, we concluded that the TGA from feedback trials was constrained merely by the physical presence of the target objects. Finally, we compared these results with those from previously published judgment studies in which the metric shapes of the very same objects had been judged. The results were the same. All studies exhibited low slopes of about .7 for the relation between actual aspect ratios and the aspect ratios obtained from judgments of grasping. We concluded that feedforward grasping is poorly guided with respect to metric object shape.

Given this conclusion, the next issue was to determine how effective grasping is performed. We hypothesized that online guidance (closed-loop control) is needed to preshape the hand with respect to object shape for effective grasps because haptic feedback without vision of the hand fails to correct the inaccuracy of perceived shape. In the last experiment, we compared the results of grasping performance with and without online guidance. In the closed-loop condition, the observers were able to see both the target objects and their hands, so they could be directly compared and the fingers could be visually guided using the perceived relation between the hand and the object. In the open-loop condition, the observers were able to see only the target objects, but not their hands; in all cases, however, they received accurate haptic feedback. The results confirmed our hypothesis. In the closed-loop condition, the TGA and the MGA aspect ratios varied reliably and accurately with the target aspect ratios. In the open-loop condition, the MGA aspect ratios, in particular, revealed that the small target aspect ratios were overestimated and that the large target aspect ratios were underestimated.

Shape perception is not calibrated by haptic feedback information, and poor shape perception yields inaccurate feedforward grasping. This is the reason why we need continuous online guidance to reach to grasp objects effectively and accurately.

\section{AUTHOR NOTE}

Correspondence concerning this article should be addressed to G. P. Bingham, Department of Brain and Psychological Science, Indiana University, 1101 East Tenth Street, Bloomington, IN 47405-7007 (e-mail: gbingham@indiana.edu).

\section{REFERENCES}

Bingham, G. P. (2005). Calibration of distance and size does not calibrate shape information: Comparison of dynamic monocular and static and dynamic binocular vision. Ecological Psychology, 17, 55-74.

Bingham, G. [P.], Coats, R., \& Mon-Williams, M. (2007). Natural prehension in trials without haptic feedback but only when calibration is allowed. Neuropsychologia, 45, 288-294.

Bingham, G. P., Crowell, J. A., \& TodD, J. T. (2004). Distortions of distance and shape are not produced by a single continuous transformation of reach space. Perception \& Psychophysics, 66, 152-169.

Bingham, G. P., Hughes, K., \& Mon-Williams, M. (2008). The coordination patterns observed when two hands reach-to-grasp separate objects. Experimental Brain Research, 184, 283-293.

Bingham, G. P., \& Mon-Williams, M. (2004). Visually guided reaching allows both slope and intercept of distance functions to be recalibrated without awareness. Journal of Vision, 4(8), 22a.

Bingham, G. P., ZaAl, F. Z., Robin, D., \& Shull, J. A. (2000). Distortions in definite distance and shape perception as measured by reaching without and with haptic feedback. Journal of Experimental Psychology, 26, 1436-1460.

Coats, R., Bingham, G. P., \& Mon-Williams, M. (in press). Calibrating grasp size and reach distance: Interactions reveal integral organization of reaching-to-grasp movements. Experimental Brain Research.

Coats, R., Mon-Williams, M., \& Bingham, G. P. (2004). Differences between natural and unnatural prehension are not inevitable if calibration is allowed. Journal of Vision, 4(8), 406a.

Johnston, E. B. (1991). Systematic distortions of shape from stereopsis. Vision Research, 31, 1351-1360.

KoEnderink, J. J. (1990). Solid shape. Cambridge, MA: MIT Press.

Lee, Y., Lind, M., \& Bingham, G. P. (2007). Shape perception is merely ambiguous, not systematically distorted. Journal of Vision, 7(9), 842a.

LeE, Y., Lind, M., \& Bingham, G. P. (2008). Shape perception is merely ambiguous, not systematically distorted. Manuscript submitted for publication. 
Lind, M., Bingham, G. P., \& Forsell, C. (2003). Metric 3D structure in visualizations. Information Visualization, 2, 51-57.

Mon-Williams, M., \& Bingham, G. P. (2005). Task constraints alter prehension movements qualitatively and quantitatively. Journal of $\mathrm{Vi}$ sion, 5(8), 124a.

Mon-Williams, M., \& Bingham, G. P. (2007). Calibrating reach distance to visual targets. Journal of Experimental Psychology: Human Perception \& Performance, 33, 645-656.

Mon-Williams, M., \& Bingham, G. P. (2008a). Calibration of grasp orientation (and wiggle room for errors in object orientation perception). Journal of Vision, 8(6), 301a.

Mon-Williams, M., \& Bingham, G. P. (2008b). Slam, stop, and flythrough: The coordinative timing and structure of reach-to-grasp movements varies with task. Manuscript submitted for publication.

Mon-Williams, M., Coats, R., \& Bingham, G. P. (2004). Reaching with feeling. Journal of Vision, 4(8), 411a.

Norman, J. F., \& Lappin, J. S. (1992). The detection of surface curvatures defined by optical motion. Perception \& Psychophysics, 51, 386-396.

Norman, J. F., \& TodD, J. T. (1993). The perceptual analysis of structure from motion for rotating objects undergoing affine stretching transformations. Perception \& Psychophysics, 53, 279-291.

Norman, J. F., \& TodD, J. T. (1996). The discriminability of local surface structure. Perception, 25, 381-398.

Norman, J. F., Todd, J. T., Norman, H. F., Clayton, A. M., \& McBride, T. R. (2006). Visual discrimination of local surface structure: Slant, tilt, and curvedness. Vision Research, 46, 1057-1069.

Norman, J. F., Todd, J. T., \& Phillips, F. (1995). The visual perception of surface orientation from multiple sources of optical information. Perception \& Psychophysics, 57, 629-636.

Pedhazur, E. J. (1982). Multiple regression in behavioral research:
Explanation and prediction (2nd ed.). New York: Holt, Rinehart \& Winston.

Perotti, V. J., Todd, J. T., Lappin, J. S., \& Phillips, F. (1998). The perception of surface curvature from optical motion. Perception \& Psychophysics, 60, 377-388.

Sheehan, S. R., Bingham, G. P., \& Mon-Williams, M. (2008). Task space calibration in Cartesian coordinates. Journal of Vision, 8(6), $481 \mathrm{a}$.

Tittle, J. S., \& Braunstein, M. L. (1993). Recovery of 3-D shape from binocular disparity and structure from motion. Perception \& Psychophysics, 54, 157-169.

Tittle, J. S., Todd, J. T., Perotti, V. J., \& Norman, J. F. (1995). Systematic distortion of perceived three-dimensional structure from motion and binocular stereopsis. Journal of Experimental Psychology: Human Perception \& Performance, 21, 663-678.

TodD, J. T., \& Bressan, P. (1990). The perception of 3-dimensional affine structure from minimal apparent motion sequences. Perception \& Psychophysics, 48, 419-430.

TodD, J. T., \& Norman, J. F. (1991). The visual perception of smoothly curved surfaces from minimal apparent motion sequences. Perception \& Psychophysics, 50, 509-523.

TodD, J. T., \& Norman, J. F. (2003). The visual perception of 3-D shape from multiple cues: Are observers capable of perceiving metric structure? Perception \& Psychophysics, 65, 31-47.

Todd, J. T., Tittle, J. S., \& Norman, J. F. (1995). Distortions of threedimensional space in the perceptual analysis of motion and stereo. Perception, 24, 75-86.

(Manuscript received February 7, 2007; revision accepted for publication March 5, 2008.) 